\title{
Climatological Relevance of Leading Seasonal Singular Vectors. Part I: Energy, Enstrophy and Spatio - Temporal Variability
}

\author{
A.Will, U.Harlander and W.Metz
}

\section{Zusammenfassung}

Schnell wachsende atmosphärische Rossbywellenzüge (RWT Moden) sind Lösungen der Singulärvektoranalyse (SV-analyse) der gedämpften barotropen Vorticitygleichung für NordWinter Grundströme. Bei Verwendung von 40 DJF Grundströmen treten die RWT Moden in nur vier Regionen auf der Welt auf. Ihre Ausbreitungspfade sind bemerkenswert konstant für Entwicklungszeiten bis zu 96h und streuen für längere Zeiten.

Die am schnellsten wachsende RWT Mode breitet sich (innerhalb von 4 Tagen) in der Region des Nordpazifiks (NPAC) aus für jeden der betrachteten DJF Grundströme. Wir bezeichnen sie deshalb als NPAC Mode. Klimatologisch hängt ihr Ausbreitungspfad vom Grundstrom ab und kaum von der jeweiligen Anfangsstruktur. Der Wachstumsfaktor der NPAC mode beträgt zwischen 10 und 20 für fast alle Grundströme und erreicht 30 für einige wenige. Es gibt viele Anfangsstrukturen, die sich auch zu einer NPAC Mode entwickeln. Ihre Wachstumsfaktoren sind jedoch kleiner. Es konnte gezeigt werden, daß die NPAC Moden mit moderaten Wachstumsfaktoren auch näherungsweise Lösungen der nichtlinearen Modellgleichung sind. Hierzu wurde eine neue Formulierung des Advektionstermes verwendet, die im Rahmen der Energie-Wirbel-Theorie abgeleitet wurde.

Die Konstanz der Ausbreitungspfade, die großen Wachstumsfaktoren und die Gültigkeit der Linearisierungsannahme für Entwicklungszeiten bis zu 96h machen die NPAC Mode zu einer dynamischen Struktur, die wichtige Teile der großräumigen interannuellen Variabilität in der Region des Nordpazifiks erklären könnte.

\footnotetext{
Abstract

Fast growing atmospheric Rossby wave trains (RWT modes) are solutions of a Singular Vector (SV) analysis of a damped barotropic vorticity equation for northern winter basic flows. Using 40 DJF basic flows RWT modes are found over four regions of the globe only. Their propagation paths are remarkably constant for development times up to $96 \mathrm{~h}$, but spread for longer times.

The fastest growing RWT mode develops over the North-Pacific (NPAC) region (within 4 days) for each of the observed DJF basic flows considered. Therefore this mode is referred to as NPAC-mode. Climatologically its development path is dominated by the basic flow
} 
rather than its particular initial structure. The growth factors of the NPAC mode range from 10 to 20 for nearly all of the basic flows. In some exceptional years they are close to 30. There exist several initial structures developing to an NPAC mode. However, they have smaller growth factors. The NPAC modes with moderate growth factors could be shown to be approximately solutions of the nonlinear model equation too. Hereto a new formulation of the advection term provided by the energy-vortex theory was used.

It appears that the constancy of propagation paths, large growth factors and the validity of the linearisation assumption up to development times of $96 \mathrm{~h}$ make the NPAC mode a candidate to explain substantial parts of large scale interannual atmospheric variability in the North Pacific region.

\section{Introduction}

The discussion of interannual to interdecadal atmospheric variability is still rather broad. Important aspects of the variability like the Pacific North America teleconnection remind on local Rossby wave trains. The letter have been shown to be solutions of theoretical and empirical linear models.

In this context the discussion of relevant mechanisms spans from the excitation of Rossby wave trains (RWTs) by local orographic and thermal forcing (Hoskins and Karoly, 1981) to RWT propagation in zonally asymmetric basic flows (Simmons et al., 1983; Hoskins and Ambrizzi, 1997; Branstator, 2002). For the latter, possible mechanism are barotropic and baroclinic instability, non-modal growth of Neutral Vectors (Metz, 1994; Goodman and Marshall, 2002) and of Singular Vectors (SVs) (Li and Ji, 1997).

SVs can be characterised as optimally growing perturbations in a finite time interval $\left[0, \mathrm{t}_{\mathrm{opt}}\right]$. They can be calculated for different linear models, basic flows, and optimisation times. Which SVs (if any) explain important phenomena on long time scales is still under discussion. In the present paper we aim to contribute to this discussion.

As pointed out by Buizza and Palmer (1995) and stated by Hoskins et al. (2000), RWTs are closely related to SVs. Together with the fact that RWTs are also important for the organisation of important aspects of interannual variability it appears obvious to try to connect both. Borges and Sardeshmukh (1995) investigated a damped barotropic model and showed that even under realistic damping SVs strongly growing within some days exist. It is also well known that leading SVs of a damped barotropic model for northern winter $300 \mathrm{hPa}$ basic flows and optimisation times of approximately 4 to 7 days evolve into RWTs with shapes similar to the PNA pattern (see e.g. Borges and Hartmann, 1992; Borges and Sardeshmukh, 1995). In addition Li and Ji (1997) investigated a damped barotropic model with an additional forcing term. They used a climatological basic flow computed from DJF 1980-1989 and calculated optimally forced SVs assuming the energy of the perturbation at initial time to be zero. The two leading optimally forced SVs of their analysis are similar to the leading SVs of the unforced equation. This indicates that the shapes and development paths of leading SVs of a damped barotropic model are rather independent of additional forcing.

Winkler et al. (2001) calculated SVs of an empirical model, developed from 7-day smoothed 
streamfunction and tropical diabatic heating DJF anomalies. The development of the leading SVs of this empirical model depends strongly on the tropical heating and provides maximal growth for optimisation times of approximately three weeks. Its nearly stationary shape showing a meridional dipole in the North Pacific reminds on a baroclinic Rossby wave also found by Klingspohn (2000) by a Multiple Singular Spectrum Analysis. Interestingly, the third pair of SVs of the empirical model develops a RWT similar to the leading SVs of the damped barotropic model independent of the tropical heating. It reaches maximal growth for optimisation times of 8 days.

As summarised above, climatologically relevant SVs have been calculated for seasonal to climatological and stationary basic flows and optimisation times of several days to weeks. As pointed out by Sardeshmukh et al. (1997) the similarity of the shapes of observed RWTs and leading SVs is not a proof of the suggestion that the SVs explain the observed RWTs. The growth and propagation velocity of the observed RWTs have to be compared with the theoretical values also. From theoretical point of view, the validity of the assumptions underlying the SV analysis for such long optimisation times is questionable.

In this and in the forthcoming paper we aim to contribute to the discussion of climatological relevance of SVs of a damped barotropic vorticity equation at $300 \mathrm{hPa}$. In comparison to empirical models, which are capable to give an efficient description of observations, the results of theoretically based models are more appropriate for physical interpretation. We concentrate on the leading SVs of the North winter, which have been shown to develop Rossby wave trains with climatologically relevant shapes rather independent of different additional forcing terms for optimisation times of 3 to 7 days.

In this paper we discuss in section 2 the basic assumptions of the SV analysis with emphasis on the linearisation assumption. We analyse the validity of the linearisation using a new formulation of the vorticity advection depending on the conserved quantities energy and enstrophy. In section 4 we discuss the properties of the leading SVs for 40 DJF basic flows providing theoretical evidence for the climatological relevance of the leading SVs.

\section{Model and Method}

Our analysis is based on the damped barotropic vorticity equation

$$
\frac{\partial \zeta}{\partial t}=-\mathcal{J}(\psi, \zeta+f)-\gamma \zeta-K \nabla^{4} \zeta
$$

Here $\psi$ denotes the streamfunction and $\zeta$ the vorticity field in $300 \mathrm{hPa}$. The linear damping term $\gamma \zeta$ is a parameterisation of the baroclinic effect of Ekman pumping and $K \nabla^{4} \zeta$ is a hyper-diffusion parameterisation of the neglected contribution of subscale interaction to the resolved scales (Reynolds-term). For our purpose, it is a reasonable model for synoptic dynamics on the $300 \mathrm{hPa}$ level. We used the same model parameters $\gamma=1 / 7 \mathrm{~d}$ and $K=$ $2.239 \cdot 10^{16} \mathrm{~m}^{4} \mathrm{~s}^{-1}$ as in Li and Ji (1997). $\mathcal{J}$ denotes the Jacobian operator.

The basic flows used to calculate the SVs are derived from 0h streamfunction of the NCEP reanalysis data $\Psi_{o}(i, j)$ on the $300 \mathrm{hPa}$ level. Here $i$ denotes the 90 days in each of the DJF seasons $58 / 59(j=58)$ to $97 / 98(j=97)$ considered. The DJF averages $\overline{\psi_{o}}(j)$ are used as 
basic flows. The index ( ) denotes observations. Due to the linear damping used (Lau, 1979) all basic flows considered are barotropically stable.

In general $\bar{\zeta}_{o}$ is not an exact solution of (1). We introduce a correction term $\mathcal{R}_{3}=-\mathcal{F}_{\overline{\zeta_{o}}}$ and obtain:

$$
\frac{\partial \overline{\zeta_{o}}}{\partial t}=-\mathcal{J}\left(\overline{\psi_{o}}, \overline{\zeta_{o}}+f\right)-\left(\gamma+K \nabla^{4}\right) \overline{\zeta_{o}}-\underbrace{\mathcal{F}_{\overline{\zeta_{o}}}}_{\mathcal{R}_{3}}
$$

Inserting $\psi=\overline{\psi_{o}}+\psi^{\prime}$ in (1), using (2) and neglecting $\mathcal{R}_{3}=-\mathcal{F}_{\overline{\zeta_{o}}}$ we obtain the following barotropic vorticity perturbation equation:

$$
\frac{\partial \zeta^{\prime}}{\partial t}=\underbrace{-\mathcal{J}\left(\psi^{\prime}, \overline{\zeta_{o}}+f\right)-\mathcal{J}\left(\overline{\psi_{o}}, \zeta^{\prime}\right)-\gamma \zeta^{\prime}-K \nabla^{4} \zeta^{\prime}}_{R_{1}}-\underbrace{\mathcal{J}\left(\psi^{\prime}, \zeta^{\prime}\right)}_{R_{2}}
$$

We neglected $R_{2}$ in (3) and solved the remaining linearised perturbation equation:

$$
\frac{\partial \zeta^{\prime}}{\partial t}=-\mathcal{J}\left(\psi^{\prime}, \overline{\zeta_{o}}+f\right)-\mathcal{J}\left(\overline{\psi_{o}}, \zeta^{\prime}\right)-\gamma \zeta^{\prime}-K \nabla^{4} \zeta^{\prime}
$$

\subsection{Singular Vector Analysis}

Equation (4) is solved in spectral space using the transform method on a T21 grid. The state vector $\boldsymbol{\Psi}(t)$ has dimension 480 and is made of the spherical harmonics coefficients. The solution of (4) is given by

$$
\boldsymbol{\Psi}(t)=\mathbb{P}_{j}(t) \Psi(0)
$$

The linear operator $\mathbb{P}_{j}(t)$ is called propagator and depends on the basic flow $\overline{\zeta_{o}}$ chosen.

The first Singular Vector $\mathbf{s}_{1}(t)$ is that solution of (4), which maximally grows in a given period of time $t_{\text {opt }}$ with respect to the scalar product $(\Psi, \Psi)_{\mathcal{S}}$ (see Borges and Hartmann (1992), Buizza and Palmer (1995), Li and Ji (1997) and Ehrendorfer and Tribbia (1997) for details). The choice of kinetic energy scalar product $(\mathcal{S}=\mathcal{H})$ is motivated by a result of turbulence theory: From dimensional arguments an upscale energy cascade is expected in a 2D turbulent flow (see Boer and Shepherd, 1983; Buizza and Palmer, 1995). The kinetic energy scalar product is given as:

$$
\mathcal{H}(t)=\|\mathbf{\Psi}(t)\|_{\mathcal{H}}^{2}=(\boldsymbol{\Psi}(t), \boldsymbol{\Psi}(t))_{\mathcal{H}}=\Psi^{T}(t) \mathbb{D} \Psi(t)
$$

The diagonal matrix $\mathbb{D}$ depends on the total wavenumber $n$ of the spherical harmonics and contains the coefficients $n(n+1)$. Assuming the energy at initial time $\|\boldsymbol{\Psi}(0)\|_{\mathcal{H}}^{2}=1$ the solution of the optimisation problem is given by the solution of the following eigenvalue equation (see Borges and Hartmann (1992)):

$$
\left(\mathbb{P}^{T}\left(t_{\text {opt }}\right) \mathbb{D} \mathbb{P}\left(t_{\text {opt }}\right)-\sigma_{k}^{2} \mathbb{D}\right) \mathbf{s}_{k}(0)=0
$$


Using (5) the development of the $\mathrm{SV} \mathbf{s}_{k}(0)$ in time can be calculated. The real eigenvalues $\sigma_{k}^{2}=\left\|\mathbf{s}_{k}\left(t_{\text {opt }}\right)\right\|_{\mathcal{H}}^{2} /\left\|\mathbf{s}_{k}(0)\right\|_{\mathcal{H}}^{2}$ are the growth factors of the kinetic energy of the SVs after $t_{\text {opt }}$. They are ranked in terms of magnitude (i.e., the first SV has maximal energy growth). In general, the SVs and the eigenvalues depend on the optimisation time chosen. Here, we present results for $t_{\text {opt }}=96 \mathrm{~h}$ only.

The SVs form a complete orthogonal basis for $t=0$ and $t=t_{\text {opt }}$. For other development times $t$ the energy scalar product of two different SVs is in general not zero $\left(\left(\mathbf{s}_{k}(t), \mathbf{s}_{l}(t)\right)_{\mathcal{H}} \neq 0\right)$. The SVs at initial time $\mathbf{s}_{k}(0)$ are normalised to have kinetic energy $\left\|\mathbf{s}_{k}(0)\right\|_{\mathcal{H}}=1$.

\section{Basic Assumptions of Singular Vector Analysis}

In the present paper we discuss the properties of the two leading SVs denoting this vorticity disturbance by $\zeta^{\prime}$. However, it can not explain the total disturbance vorticity field of the atmosphere. The nonlinear interaction of $\zeta^{\prime}$ with the remaining atmospheric disturbances $\zeta^{\prime \prime}$ will be discussed in the second part of the paper. The validity of the assuptions $\mathcal{R}_{2} \ll \mathcal{R}_{1}$ and $\mathcal{R}_{3} \ll \mathcal{R}_{1}$ in (4) depends on $\overline{\zeta_{o}}$ and $\zeta^{\prime}$. It will be discussed in the following.

The term $\mathcal{R}_{3}$ may be regarded as a low frequency additional forcing term in (4) and may be assumed constant on the time scale of SV development. It is unknown within the model considered and can not be discussed theoretically. Howevere, there is some empirical evidence for neglecting $\mathcal{R}_{3}$ introduced in (2) in comparison to $\mathcal{R}_{1}$ introduced in (3) analysing the two leading SVs of DJF basic flows. Figure 1 shows the leading SV of eq.(4) for DJF basic flow of season $j=78$. It is similar to the optimally forced leading SV presented by Li and Ji (1997) and to the third SV of the empirical linear model presented by Winkler et al. (2001) (already discussed in section 1), which is nearly independent of the SST forcing considered. The shape and propagation path of the leading SVs seem to be rather independent of the low frequency forcing term $\mathcal{R}_{3}$.

Usually the validity of a linearised model is limited by the classical limit of linearisation given by the time at which the nonlinear term of the model becomes important for the development of the initial disturbance. At that time we expect a divergence of the trajectories calculated by the linearised and the nonlinear model. In numerical experiments with SVs the optimisation time has to be chosen close to the classical limit of linearisation.

It is well known that the SVs of forecast models efficiently describe the development of the forecast error for optimisation times between 24 and 48 h (see Buizza and Palmer (1995), Ehrendorfer and Tribbia (1997) and Coutinho and Hoskins (2004) for details). Initial amplitudes larger than observed or longer optimisation times violate the linearisation condition. As will be shown in more detail in the second part of the paper, we found the same for the leading SVs of the damped barotropic model and DJF basic flows. At longer development times the nonlinear term is not one order of magnitude smaller than the linear terms anymore and this cannot be neglected.

The classical limit of linearisation is not relevant if the disturbance may be shown to be a solution of the linear and of the nonlinear model as well. We name such disturbances free disturbances. It is well known, that the zonally propagating Rossby-Haurwitz waves 
$\psi^{\prime}(\lambda, \varphi)=\psi_{k l} \cos (l \varphi) \cos (k \lambda-\omega t)$ with arbitrary constant amplitudes $\psi_{k l}$ are free disturbances of the disturbance barotropic vorticity equation on the beta plane with a zonal basic flow $\bar{\psi}(\varphi)=\psi_{0}-m \varphi$ having a constant meridional gradient of the streamfunction field.

In general, a disturbance field $\zeta^{\prime}$, which solves the linearised equation (3) is a free disturbance if the disturbance voriticity $\zeta^{\prime}$ may be written as a function of the disturbance streamfunction $\psi^{\prime}$ (see Branstator and Opsteegh (1989) and Lange (2002, p.557) for details):

$$
\zeta^{\prime}=f\left(\psi^{\prime}\right) \Rightarrow \mathcal{J}\left(\psi^{\prime}, \zeta^{\prime}\right)=0
$$

For Rossby-Haurwitz waves on the beta plane the relation reads

$$
\zeta^{\prime}=-\left(k^{2}+l^{2}\right) \psi^{\prime}
$$

In practice it is rather difficult to find free disturbances for realistic basic flows. However, (8) may be used to analyse the functional dependence of vorticity and streamfunction of a disturbance being solution of the linearised model equation. In the case of SVs, which have a time dependent shape and amplitude a more suitable formulation of (8) may be used, resulting from the energy-vortex theory (Névir, 2004). For the barotropic vorticity equation it has been shown by Névir and Blender (1993) (see also Lange, 2002, p.544), that the Jacobian in (1) may be written as

$$
\begin{aligned}
\mathcal{J}\left(\psi, \zeta_{a}\right) & =-\mathcal{J}\left(\zeta_{a}, \psi\right)=\mathcal{J}\left(\frac{\partial \mathcal{E}}{\partial \zeta_{a}}, \frac{\partial \mathcal{H}}{\partial \zeta_{a}}\right) \\
\text { with } \quad \frac{\partial}{\partial \zeta_{a}} \mathcal{E}\left[\zeta_{a}\right] & =\frac{\partial}{\partial \zeta_{a}}\left[\iint_{\Omega} \frac{1}{2} \zeta_{a}^{2} d \Omega\right]=\zeta_{a} \\
\text { and } \quad \frac{\partial}{\partial \zeta_{a}} \mathcal{H}\left[\zeta_{a}\right] & =\frac{\partial}{\partial \zeta_{a}}\left[-\iint_{\Omega} \frac{1}{2} \psi \zeta_{a} d \Omega\right]=-\psi
\end{aligned}
$$

Here $\partial$ denotes functional derivative, [] denotes the dependence of a function and $\iint_{\Omega}$ the integral over the sphere and $\zeta_{a}=\zeta+f$ the absolute vorticity. Equation (10) conveys important new insights into barotropic dynamics: it makes evident, that the enstrophy $\mathcal{E}$ is as important as the global kinetic energy $\mathcal{H}$. They determine the conservative barotropic dynamics. Furthermore, from the antisymmetric structure of the Jacobian it follows that the term becomes zero if the kinetic energy may be written as a function of the enstrophy:

$$
\mathcal{H}(t)=f(\mathcal{E}(t)) \Rightarrow \mathcal{J}\left(\psi, \zeta_{a}\right)=0
$$

This may be seen as follows:

$$
\frac{\partial \mathcal{H}\left[\zeta_{a}\right]}{\varpi \zeta_{a}}=\frac{d f}{d \mathcal{E}} \frac{\varpi \mathcal{E}\left[\zeta_{a}\right]}{\varpi \zeta_{a}}=\frac{d f}{d \mathcal{E}} \zeta_{a}
$$

Inserting (12) in (10) we get

$$
\mathcal{J}\left(\frac{\partial \mathcal{E}}{\partial \zeta_{a}}, \frac{\partial \mathcal{H}}{\partial \zeta_{a}}\right)=\mathcal{J}\left(\zeta_{a}, \frac{d f}{d \mathcal{E}} \zeta_{a}\right)=\frac{d f}{d \mathcal{E}} \mathcal{J}\left(\zeta_{a}, \zeta_{a}\right)=0 .
$$


The term $R_{2}$ in (3) neglected in the linear model (4) has the same structure as the Jacobian in (10). Furthermore, the kinetic energy $\mathcal{H}^{\prime}=\mathcal{H}\left[\zeta^{\prime}\right]$ of the leading SVs is time dependent. This opens the possibility to analyse the much simpler relation between $\mathcal{H}^{\prime}$ and $\mathcal{E}^{\prime}=\mathcal{E}\left[\zeta^{\prime}\right]$ (depending on time only) instead of analysing the relation of $\psi^{\prime}$ and $\zeta^{\prime}$ (depending on space and time).

The most simple functional dependence of $\mathcal{H}^{\prime}$ and $\mathcal{E}^{\prime}$ is their linear dependence:

$$
\mathcal{H}^{\prime}(t)=a+b \mathcal{E}^{\prime}(t) \Rightarrow \frac{\partial \mathcal{H}^{\prime}}{\partial \zeta^{\prime}}=b \frac{\partial \mathcal{E}^{\prime}}{\partial \zeta^{\prime}} \Rightarrow-\psi^{\prime}(\mathrm{x}, \mathrm{t})=\mathrm{b} \zeta^{\prime}(\mathrm{x}, \mathrm{t})
$$

From physical reasons the integration constant $a$ should be zero. Analogous to the relation (9) valid for the Rossby-Haurwitz waves the coefficient $b$ may be interpreted as a function of the dominant wavenumber $\hat{n}$ of the RWT mode. On the sphere it is adjacent to suggest: $b^{-1}=\hat{n}(\hat{n}+1)$.

The functional dependence of energy and enstrophy introduced in (14) can be analysed for all SVs exhibiting a significant dependence of energy and enstrophy on time. The enstrophy of a SV can be calculated using the enstrophy scalar product:

$$
\mathcal{E}(t)=\|\Psi(t)\|_{\mathcal{E}}^{2}=(\Psi(t), \Psi(t))_{\mathcal{E}}=\Psi^{T}(t) \mathbb{D}^{2} \Psi(t)
$$

For obvious reasons, we can not expect to find exact relationship (14) between $\mathcal{H}^{\prime}$ and $\mathcal{E}^{\prime}$. In comparison to the known free disturbances like the Rossby-Haurwitz waves on the $\beta$-plane in a constant basic flow, the SVs have time dependent amplitudes and group velocities and the basic flows considered depend on longitude and latitude in a complicated manner.

\section{Properties of Leading Singular Vectors}

In the following we discuss the properties of the leading two SVs of 40 DJF basic flows. If not stated else the optimisation time is $t_{\text {opt }}=96 \mathrm{~h}$. We denote the k-th SV of season $j$ at development time $t$ as $\mathbf{s}_{k}(t, j)$.

Fig. 1 shows the SV $\mathbf{s}_{1}(t, 78)$ at development times 0,24 and $t_{\text {opt }}=96 \mathrm{~h}$. At initial time about $70 \%$ of the SVs energy is concentrated within a Rossby wave train (RWT) with a dominant zonal wave number $k \simeq 6$ in the region $5^{\circ} \mathrm{E}$ to $140^{\circ} \mathrm{E}$ and $5^{\circ} \mathrm{N}$ to $45^{\circ} \mathrm{N}$. The other $30 \%$ of SVs energy are spread over half of the globe. At development times $24<t<96$ the shape of the leading SV reminds on a local RWT with zonal wave number $k \simeq 5$. More than $90 \%$ of the SVs kinetic energy is concentrated now within the RWT located in the region $120^{\circ} \mathrm{E}$ to $220^{\circ} \mathrm{E}$ and $24^{\circ} \mathrm{N}$ to $66^{\circ} \mathrm{N}$. The group velocity and propagation path are consistent with RWT theory (Buizza and Palmer, 1995; Hoskins et al., 2000). The second SV is in phase quadrature to the first (see also $\mathrm{Li}$ and Ji, 1997) and has a very similar eigenvalue $\left(\sigma_{1}^{2}(78)=11.8\right.$ and $\left.\sigma_{2}^{2}(78)=11.3\right)$. As will be shown in the following the pair of SVs 1 and 2 in all other seasons $j=58$ to $j=97$ have very similar properties as those of season $j=78$. We therefore name the SVs 1 and 2 "NPAC mode".

The NPAC modes of all seasons considered are located in the same region as in season $j=78$. Fig. 2 shows the dependence of the propagation path of the NPAC mode on the basic flow. It 

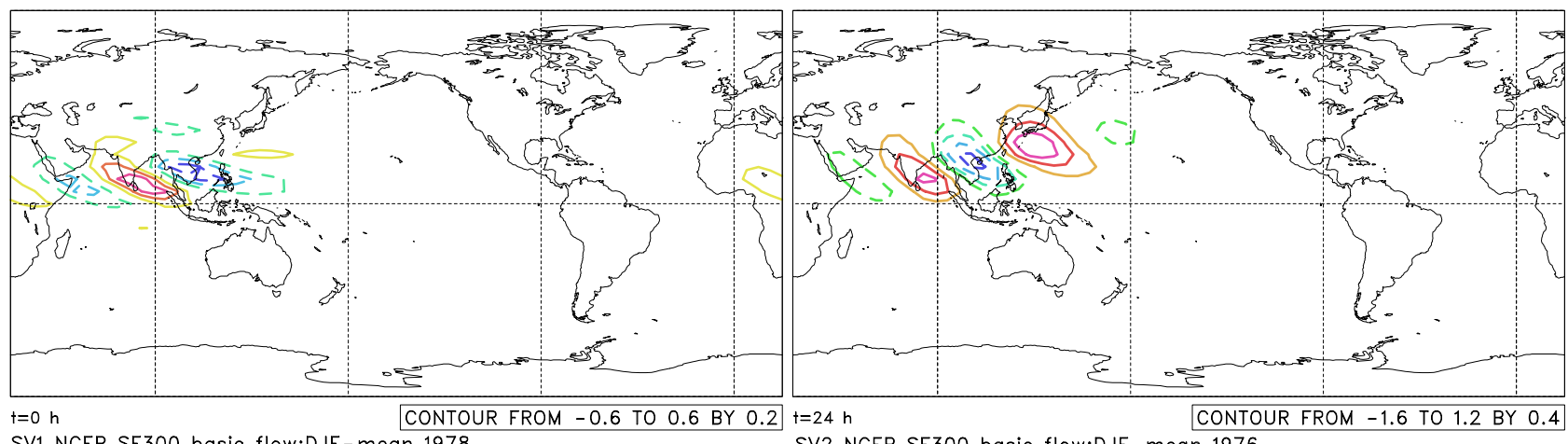

$\mathrm{t}=24 \mathrm{~h}$

CONTOUR FROM -1.6 TO 1.2 BY 0.4 SV1 NCEP SF300 basic flow:DJF-mean 1978

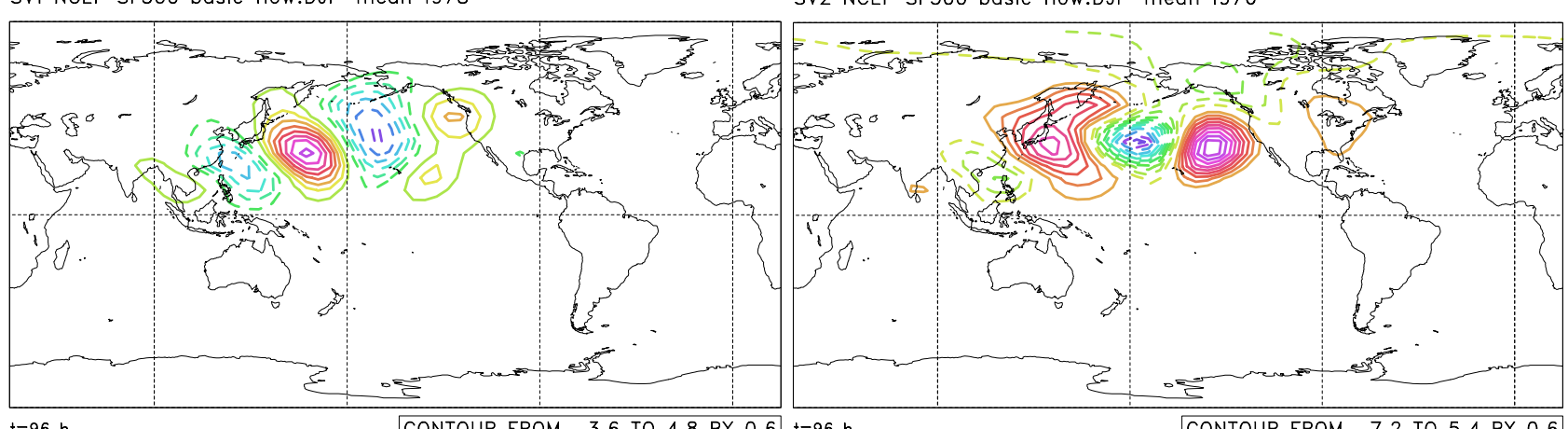

$\mathrm{t}=96 \mathrm{~h}$

CONTOUR FROM -3.6 TO 4.8 BY $0.6 \quad t=96 \mathrm{~h}$

CONTOUR FROM -7.2 TO 5.4 BY 0.6

Figure 1: First SV $\mathrm{s}_{1}(t, 78)$ for development times $t=0 \mathrm{~h}, t=24 \mathrm{~h}$ and $t_{\text {opt }}=96 \mathrm{~h}$ and DJF basic flow of season $j=78$ and the SV $\mathbf{s}_{1}(96,76)$ for $t=96 \mathrm{~h}$ and $j=76$.

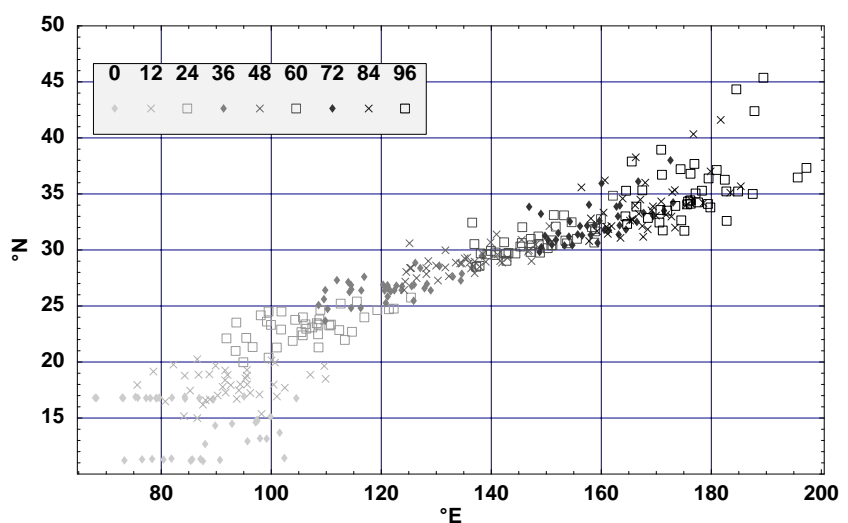

shows the centers of location of the NPAC mode for all seasons $j$ and development times up to $t=96 \mathrm{~h}$. The center of location is given by the location of the maximum of the Gaussian envelope. The parameters of the envelopes are calculated as least square fits to the kinetic energy of the wave train. The development paths of the NPAC mode for different seasons $j$ spread much more for times $t>96 \mathrm{~h}$, for which the RWT modes energy begins to dissipate (not shown). Furthermore, we investigated the sensitivity of propagation paths to initial structures calculating the development paths of $\mathbf{r}_{1, l}(t, j)(l \varepsilon(58,59,65,67,79,81,86,91))$ which is the first $\mathrm{SV} \mathrm{s}_{1}(0, l)$ of season $l$ at initial time developing in the basic flow of season $j$ (not shown). However, for $l=j$ it holds $\mathbf{r}_{1, j}(t, j)=\mathbf{s}_{1}(t, j)$. No significant differences could be found in comparison to results presented in figure 2.

The eigenvalues of the 1st and 2nd SV are close to each other for every DJF basic flow and range for different seasons $j$ from 10 to 30 . Fig.3 shows the eigenvalues of SV 1 $\sigma_{1}^{2}(j)$. The growth of the NPAC mode is dominated by the basic flow and by initial structures. Figure 3 shows the eigenvalues $\sigma_{1}^{2}(j)$ in comparison to the growth factors $g=$ 


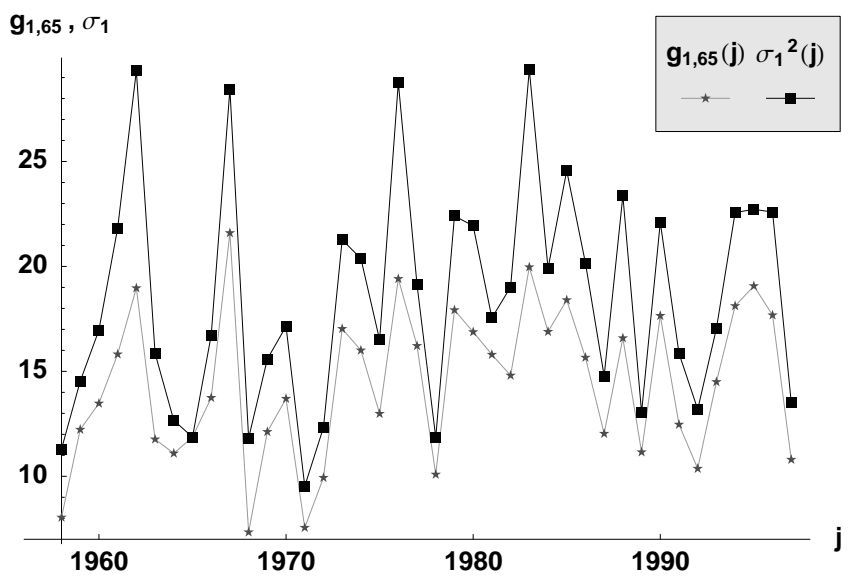

Figure 3: Eigenvalues $\sigma_{1}^{2}(j)$ and the growth factor $g_{1,65}(j)$ for all seasons $j \cdot g_{1,65}(j)$ is the growth of $\mathbf{s}_{1}(0,65)$ in the basic flow of season $j$.

$\left\|\mathbf{r}_{1,65}(96, j)\right\| /\left\|\mathbf{r}_{1,65}(0, j)\right\|$ of the first SV of season $l=65$ developing in all seasons $j$. The high correlation between both time serieses shows the influence of the basic flow. The quotient of the eigenvalues and growth factors $g_{1,65}$ ranges between 1 and 2 and exhibits the influence of initial structures.

The shape of the NPAC mode at a given development time $t$ is rather constant for development times up to $96 \mathrm{~h}$. The first SV $\mathbf{s}_{1}(96,78)$ of season $j=78$ is rather typical. It is also similar to the first SV of the climatological basic flow $\widetilde{\psi}$, to the leading SV presented by $\mathrm{Li}$ and Ji (1997) and to the third SV presented by Winkler et al. (2001) calculated for much longer optimisation times.

The shape of the NPAC mode depends weakly on the natural variability of the basic flow and practically not on the natural variability of the initial structures. We found the most significant differences in shape for SVs with maximal eigenvalues. One example is the first $\mathrm{SV}$ of season $j=76$ (also shown in Fig. 1) with $\sigma_{1}^{2}(76)=28.8$. The stronger influence of the basic flow than of initial structures on the shape is shown by significantly higher correlation of $\mathbf{s}_{1}(t, 76)$ with $\mathbf{r}_{1,78}(t, 76)$ (not shown) for development times $24 \leq t \leq 96 \mathrm{~h}$ than of the SVs $\mathrm{s}_{1}(t, 78)$ with $\mathbf{s}_{1}(t, 76)$.

In all seasons considered there exist just four other pairs of SVs with properties comparable to the NPAC mode but located at different spots of the globe. They will be discussed elsewhere. ${ }^{1}$ The other SVs (out of 480 for each basic flow) do not develop a growing, localised and propagating wave train with a decreasing dominant wave number.

The properties of the NPAC mode presented make it a candidate to explain important parts of interannual to interdecadal atmospheric variability in the region of the North Pacific. Any disturbance field existing in the region of $\mathbf{s}_{1}(0, j)$ develops an NPAC mode with a path and shape typical for the basic flow in the linear model. Its growth and propagation velocity may be expected to be dominated by the basic flow and disturbed by the variability of the initial disturbance field.

The properties of the NPAC mode presented do not answer the question, whether the NPAC mode may be expected to occur in the real atmosphere. However, this should be the case if the assumptions of the SV analysis are valid for the NPAC mode. In the following we analyse the validity of the linearisation assumption.

As shown in (10) the enstrophy and the kinetic energy of the motion determine the conservative barotropic dynamics. The disturbance enstrophy $\mathcal{E}^{\prime}$ and disturbance energy $\mathcal{H}^{\prime}$ determine

\footnotetext{
${ }^{1}$ Examples are given at www.uni-lepzig.de/ meteo/AWILL/anima.html.
} 

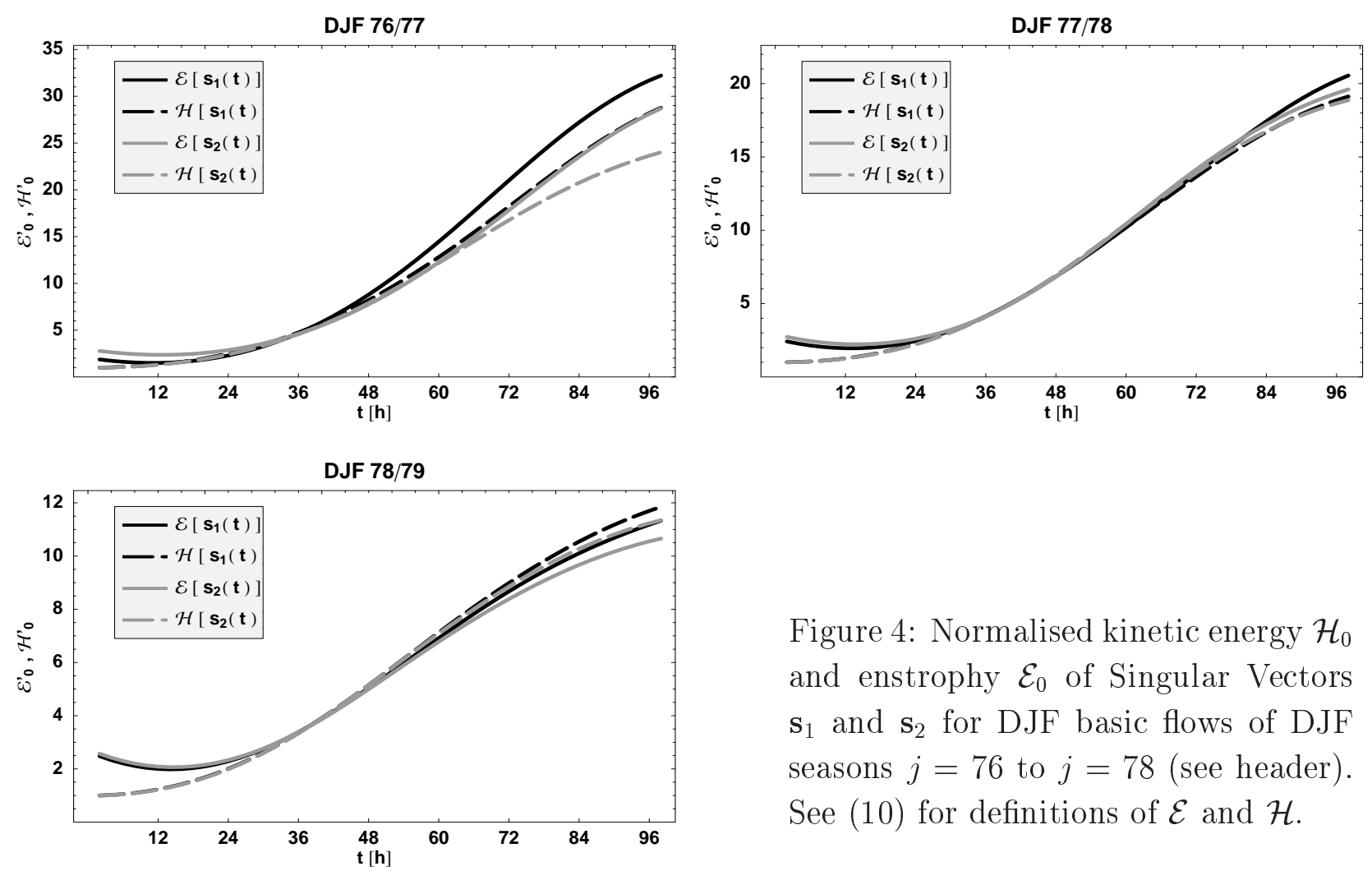

Figure 4: Normalised kinetic energy $\mathcal{H}_{0}$ and enstrophy $\mathcal{E}_{0}$ of Singular Vectors $\mathbf{s}_{1}$ and $\mathbf{s}_{2}$ for DJF basic flows of DJF seasons $j=76$ to $j=78$ (see header). See (10) for definitions of $\mathcal{E}$ and $\mathcal{H}$.

the nonlinear interaction of the disturbance. Fig.4 shows the development of kinetic energy and enstrophy of SVs 1 and 2 for seasons $j=76$ to $j=78$. The energy is normalised to one at initial time and the enstrophy is normalised in such a way, that at $t=36 \mathrm{~h}$ the normalised kinetic energy and the normalised enstrophy have the same values. The kinetic energy is strictly monotonic increasing, while the enstrophy decreases at the beginning. It reaches its initial value after approximately 30h (first period) and develops hereafter very similar to the energy (second period). The flow of energy and of enstrophy to the large scales during the first period comes along with a loss of enstrophy. Significant differences are found between the developments of energy and enstrophy of the leading SVs of seasons $j=76$ and $j=78$ during the second period of development. In season $j=78$, for which the eigenvalue is $\sigma_{1}^{2}(78)=\mathcal{H}\left[s_{1}(96,78)\right]=11,8$ the enstrophy growth is very similar to the energy growth. The leading SVs of $j=76$ having high eigenvalues $\left(\sigma_{1}^{2}(76)=\mathcal{H}\left[s_{1}(96,76)\right]=28,8\right)$ exhibit a significantly stronger growth of enstrophy. This may be understood qualitatively as a pirouette effect known from figure skating. The figure skater tucks up his legs increasing his kinetic energy and his angular velocity. In hydrodynamics the basic flows wave guiding effect contracts the volume of the SV. This increases its vorticity even if the kinetic energy remains constant. The work done by the basic flow on the SV increases the kinetic energy and enstrophy of the SV. This effect is confirmed by direct comparison of SVs at optimisation time of seasons $j=78\left(\sigma_{1}^{2}(78)=11.9\right)$ and $j=76\left(\sigma_{1}^{2}(76)=28.8\right)$ shown in fig.1. Here the diameter of the vortex is smaller for $j=76$ than for $j=78$. The behavior of the enstrophy in season $j=76$ could be found in all other seasons $j$ with high eigenvalues $\left(\sigma_{1}^{2}(j) \geq 20\right)$.

We investigated the linear dependence of $\mathcal{H}\left[s_{i}(t, j)\right]$ and enstrophy $\mathcal{E}\left[s_{i}(t, j)\right]$ introduced in (14)) of the SVs 1 and 2 for all DJF basic flows considered. In Fig.5 we show the inverse of the normalised coefficients $b_{i}(t)$ in dependence of the development time of the NPAC mode for seasons $j=76$ to $j=78$. Here $b_{1}$ and $b_{2}$ denote the coefficients for $\mathbf{s}_{1}(t, j)$ and $\mathbf{s}_{2}(t, j)$. The coefficient of the sum $\left(\mathbf{s}_{1}(t, j)+\mathbf{s}_{2}(t, j)\right)$ is denoted by $b_{12}$. Two periods may be identified. In 

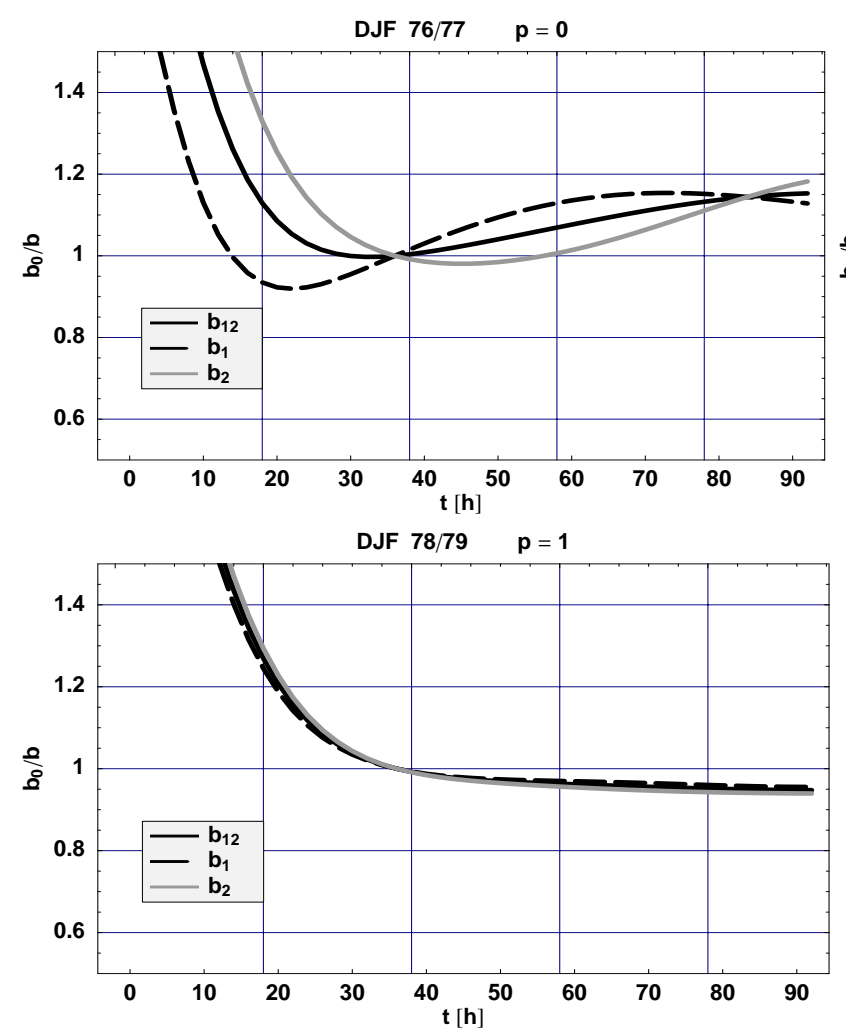

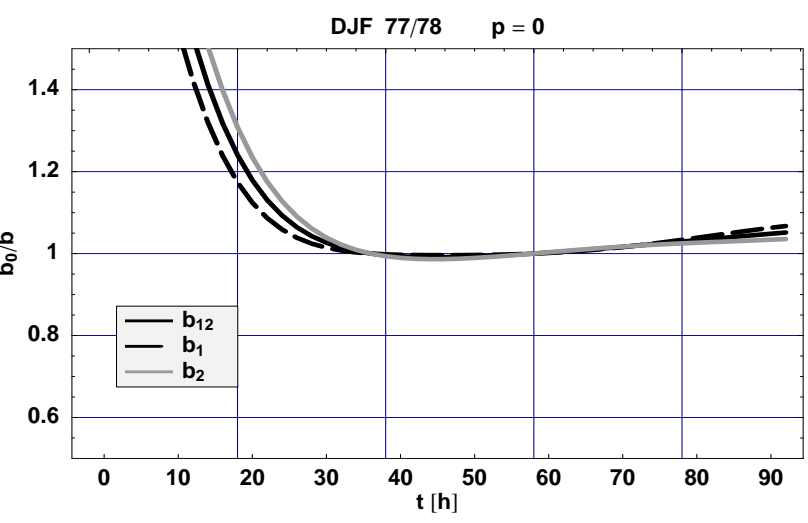

Figure 5: Normalised inverse coefficients $b_{0} / b_{i}$ of $\mathrm{SVs} \mathrm{s}_{1}, \mathbf{s}_{2}$ and $\mathbf{s}_{12}=\mathbf{s}_{1}+\mathbf{s}_{2}$ for seasons $j=76$ to $j=78$ (see header). $p=1$ means that the standard deviation of $b_{i}$ in the time span $36 h<t<96 h$ is less than $2 \%$ of the mean. See (14) for definition of the coefficient $b$.

the first period $(t<36 \mathrm{~h})$ a strong increase of $b_{i}$ is observed (notice that the inverse normalised coefficient is shown in Fig.5). It describes the decrease of the dominant wavenumber of the NPAC-mode. In the second period $(36 \mathrm{~h}<t<96 \mathrm{~h})$ we found a moderate time dependence of $b_{i}$. Following (13) this indicates that the NPAC mode is approximately a solution of the (nonlinear) barotropic vorticity perturbation equation (3) too. The results for seasons $j=76$ to $j=78$ shown in Fig. 5 represent three typical cases. Season $j=78$ reveals coefficients $b_{0} / b_{1}(t)=b_{0} / b_{2}(t)=0.92 \pm 0.02$, which are nearly constant. Investigating 40 seasons we found 14 exhibiting nearly constant coefficients $b_{i}$ in the second period. This property is noted in the header of the figure by $p=1$. In season $j=77$ we still find $b_{1}(t) \simeq b_{2}(t)$ with a moderate decrease of the coefficient of $5 \%$. Season $j=76$ exhibits a strong time dependence of the coefficients $b_{1}$ and $b_{2}$. The same was found for the coefficients of the leading SVs of the other 3 seasons with high eigenvalues $\left(\sigma_{1}^{2}(j) \geq 20\right)$.

The means $\widehat{b_{i}(j)}$ of the coefficient $b_{i}(t, j)$ over the second period $(36 \leq t \leq 96)$ exhibit a high correlation $c$ with the eigenvalues of the leading SVs $\left(c\left(\sigma_{12}^{2}, \widehat{b_{12}}\right)=-0.75\right)$. High eigenvalues co-variate with dominant wavenumbers. This result is consistent with the discussion of the development of energy/enstrophy and the pirouette effect of the wave guide.

The NPAC mode could be identified to be nearly a free solution of the damped barotropic vorticity equation during the second period of development for a substantial part of the DJF basic flows cosidered. Together with the classical limit of linearisation being valid during the first period of development (see section 3) the results justify the linearisation assumption for development times up to $96 \mathrm{~h}$ for many of the basic flows considered. It may therefore be expected to find the NPAC mode in observations as if the growth factor is expected to show a substantial variability. 


\section{Summary and Conclusions}

The leading SVs of the damped barotropic vorticity equation for North winter seasonal and climatological basic flows are known to develope Rossby wave trains in the North Pacific region well observed in the atmosphere for optimisation times of several days to weeks. However, it is still an open question, whether such SVs can explain these observations. We address the question in two steps. In this paper we examined the space-time variability of the leading SVs for 40 DJF basic flows on $300 \mathrm{hPa}$ level derived from NCEP data in more detail than has been done before. Additionally the underlying linearisation assumption of the SV analysis has been investigated. In a forthcoming paper, the calculated space-time variability is compared with observations.

It was found that the two leading SVs (NPAC mode) for each basic flow are in phase quadrature and exhibit a remarkably constant propagation path in the North Pacific region for development times of $96 \mathrm{~h}$ independent of the natural variability of the basic flow and small changes of initial structures. They exhibit remarkable energy growth factors of 10 to 30 within 96h. This makes the NPAC mode a candidate to explain important parts of interannual to interdecadal atmospheric variability.

Beyond the development time of $96 \mathrm{~h}$ we found a spread of propagation paths and of the shape of the Rossby wave trains for all of the 40 DJF seasonal basic flows considered indicating the end of the Rossby wave guide. We therefore concentrated our analysis on the NPAC mode for optimisation times up to $96 \mathrm{~h}$. This time has to be regarded as model dependend. Outside of the wave guide neglected processes like baroclinic instability and nonlinear interaction become important for further development of the Rossby wave trains.

The shape of the NPAC mode could be shown to be practically independent of small changes of initial structures. However, four extremely growing NPAC modes $\left(\sigma^{2}>20\right)$ exhibit significantly increased wave numbers and small changes of the shapes (see Fig.1). Small changes of initial structures diminish the growth factor up to a factor of two. We therefore expect a substantial spread of the growth factors in observations due to the spatial variability of initial structures.

It could be shown that the leading SVs (NPAC mode) are approximately solutions of the nonlinear equation for development times up to $96 \mathrm{~h}$ for the most of the basic state flows considered. Hereto a new formulation of the nonlinear term was used (Névir and Blender, 1993) depending on energy and enstrophy of the vorticity field. We found two periods of development of the NPAC-mode. During the first period of development (approx. 36h) the NPAC mode develops a Rossby wave train with increasing energy and wave length accompanied by loss of enstrophy. Assuming amplitudes near statistical expectation value at initial time, the classical limit of linearisation is reached at the end of this period of development. During the second period of development the NPAC mode is still propagating but shape preserving and its energy and enstrophy are growing with nearly equal rates. We found approximately a linear dependence of disturbance kinetic energy and disturbance enstrophy for development times $36<t<96$. This characterizes the leading SVs approximately as solutions of the nonlinear model perturbation equation also, which we name free disturbances. This is remarkable since up to now no freee disturbances with time dependent energy could 
be found. What is most important now, it may be expected to find these free disturbances in observations. Other disturbances do not reach sufficiently large amplitudes to become dominant in the atmosphere and/or do not have a unique propagation path.

Notice that the limit of validity of the linearisation coincides with the predictability limit of the model used and may be regarded as model dependent too.

However, even if it may be expected to find the NPAC mode in observations it is still an open question due to further assumptions of the model. This is investigated in the second part of the paper.

\section{References}

Boer, G. and Shepherd, T. (1983). Large-Scale Two-Dimensional Turbulence in the Atmosphere. Journal of the Atmospheric Sciences, 40, 164-184.

Borges, M. D. and Hartmann, D. L. (1992). Barotropic instability and optimal perturbations of observed nonzonal flows. Journal of the Atmospheric Sciences, 49(4), 335-354.

Borges, M. D. and Sardeshmukh, P. D. (1995). Barotropic Rossby Wave Dynamics of Zonally Varying Upper-Level Flows During Northern Winter. Journal of the Atmospheric Sciences, 52(21), 3779-3796.

Branstator, G. (2002). Circumglobal Teleconnections, the Jet Stream Waveguide, and the North Atlantic Oscillation. Journal of Climate, 15, 1893-1910.

Branstator, G. and Opsteegh, J. (1989). Free solutions of the barotropic vorticity equation. Journal of the Atmospheric Sciences, 46(12), 1799-1814.

Buizza, R. and Palmer, T. (1995). The Singular-Vector Structure of the Atmospheric Global Circulation. Journal of the Atmospheric Sciences, 52, 1434-1456.

Coutinho, M. and Hoskins, B. (2004). The Influence of Physical Processes on Extratropical Singular Vectors. Journal of the Atmospheric Sciences, 61, 195-209.

Ehrendorfer, M. and Tribbia, J. J. (1997). Optimal Reduction of Forecast Error Covariances through Singular Vectors. Journal of the Atmospheric Sciences, 54(2), 286-313.

Goodman, J. C. and Marshall, J. (2002). Using Neutral Vectors to Study Low-Frequency Atmospheric Variability. Journal of the Atmospheric Sciences, 59, 3206-3222.

Hoskins, B., Buizza, R., and Badger, J. (2000). The Nature of Singular Vector Growth and Structure. Quarterly Journal of the Royal Meteorological Society, 126, 1565-1580.

Hoskins, B. J. and Ambrizzi, T. (1997). Stationary Rossby-Wave Propagation in a Baroclinic Atmosphere. Quarterly Journal of the Royal Meteorological Society, 123, 919-928.

Hoskins, B. J. and Karoly, D. J. (1981). The Steady Linear Response of a Spherical Atmosphere to Thermal and Orographic Forcing. Journal of the Atmospheric Sciences, 38, 1179-1196. 
Klingspohn, M. (2000). Interdekadische Klimavariabilität über dem Nordatlantik-Statistische Analysen und Modellstudien. Wiss. Mitteilungen aus dem Institut für Meteorologie der Universität Leipzig, 21, 104pp.

Lange, H.-J. (2002). Die Physik des Wetters und des Klimas. Dietrich Reimer Verlag Berlin.

Lau, N.-C. (1979). The Observed Structure of Tropospheric Stationary Waves and the Local Balance of Vorticity and Heat. Journal of the Atmospheric Sciences, 36, 996-1016.

Li, Z. and Ji, L. (1997). Efficient Forcing and Atmospheric Teleconnections. Quarterly Journal of the Royal Meteorological Society, 123, 2401-2423.

Metz, W. (1994). Singular Modes and Low-Frequency Atmospheric Variability. Journal of the Atmospheric Sciences, 51, 1740-1753.

Névir, P. (2004). Ertel's Vorticity Theorems, the Particle Relabeling Symmetry and the Energy-Vorticity Theory of Fluid Mechanics. Meteorologische Zeitschrift, 6, 485-499.

Névir, P. and Blender, R. (1993). A Nambu Representation of Incompressible Hydrodynamics Using Helicity and Enstrophy. Journal of Physics A: Mathematical and General, 26, L1189-L1193.

Sardeshmukh, P. D., Newman, M., and Borges, M. D. (1997). Free Barotropic Rossby Wave Dynamics of the Wintertime Low-Frequency Flow. Journal of the Atmospheric Sciences, $\mathbf{5 4}(1), 5-23$.

Simmons, A. J., Wallace, J. M., and Branstator, G. W. (1983). Barotropic wave propagation and instability, and atmospheric teleconnection patterns. J. Atmos. Sci., 40, 1363-1392.

Winkler, C. R., Newman, M., and Sardeshmukh, P. D. (2001). A Linear Model of Wintertime Low-Frequency Variability. PartI: Formulation and Forecast Skill. Journal of the Atmospheric Sciences, 14, 4474-4494. 